\title{
Radical mastectomy of massive stage IV triple negative breast cancer to improve hygiene and comfort in a 90-year-old woman: a case report
}

\author{
Christopher $\mathrm{Ng}^{1,2}$, Nicole C. Jamieson ${ }^{1 \wedge}$, Richard C. Spinale ${ }^{1}$ \\ ${ }^{1}$ Department of Surgery, Garden City Hospital, Garden City, MI, USA; ${ }^{2}$ Michigan State University College of Osteopathic Medicine, East Lansing, \\ MI, USA \\ Correspondence to: Christopher Ng. Department of Surgery, Garden City Hospital, 6245 Inkster Rd, Garden City, MI 48135, USA. \\ Email: chrisngd150@gmail.com.
}

\begin{abstract}
Radical mastectomies are rarely performed today due to the advent of less invasive procedures, advances in radiation therapy and chemotherapy, and improved screening technology. The strongest indication for such a procedure is tumor fixation to the pectoralis muscles, a rare occurrence. We detail a case of a 90-year-old woman with a massive fungating right breast mass with unknown onset. Upon presentation, the patient complained of malodorous discharge from the breast mass. She denied pain, sensation of a mass in her breast, or constitutional symptoms. In fact, the patient believed the discharge was originating from a wound on the inferior surface of her breast, oblivious to the presence of a possible malignancy. However, she admitted to difficulty in caring for the open lesion, keeping herself and clothing clean and performing some of her daily activities of living. Computerized tomography (CT) imaging revealed a large, ulcerative, necrotic, and locally advanced breast lesion, invading through the skin and chest wall musculature, exhibiting obvious malignancy. Due to these findings and her poor ability to comply with chemotherapy and radiation, the decision was made to treat her disease with radical mastectomy. Post-operative pathological analysis revealed the tumor to be an advanced stage triple negative invasive ductal carcinoma with positive axillary lymph nodes. Radical mastectomy proved to be a viable treatment for local control and hygiene as she experienced a remarkably good performance status. Four months later, she presented with a cutaneous metastatic lesion on the contralateral breast and axillary lymph node metastasis. This was treated with left breast modified radical mastectomy. However, two months later, follow-up positron emission tomography (PET) CT detected hypermetabolic foci in multiple locations that were consistent with distant metastasis. The patient's circumstances presented a situation in which conventional treatment methods were not ideal. Due to her age and living condition, our management of the patient was focused on improving her quality of life as opposed to eradicating her disease. As of writing this case, the patient has admitted to dramatic improvement in her daily life, despite her disease status. In this article, we discuss the benefits and limitations of surgical and medical treatment for such rare lesions.
\end{abstract}

Keywords: Radical mastectomy; fungating; triple negative breast cancer; invasive ductal carcinoma; case report

Received: 06 June 2021; Accepted: 19 August 2021; Published: 30 June 2022.

doi: 10.21037/asj-21-45

View this article at: https://dx.doi.org/10.21037/asj-21-45

^ ORCID: Christopher Ng, 0000-0001-8815-7958; Nicole C. Jamieson, 0000-0003-2242-2098. 


\section{Introduction}

According to the American Cancer Society (ACS), 1 in $8(13 \%)$ women will be diagnosed with breast cancer in their lifetime (1). Approximately $81 \%$ of these cases can be classified as histologically invasive tumors, or neoplastic cells that have penetrated glands or ducts and grown into areas separate from their site of origin (1). The majority $(75 \%)$ of these invasive tumors are further classified as invasive ductal carcinomas (1). Other variants of invasive tumors include lobular, tubular, mucinous, papillary, and inflammatory.

In developed nations, the availability of modern screening methods enable the detection and treatment of breast cancers at early stages. However, $10-15 \%$ of breast cancers are still classified as locally advanced breast cancers (LABC) (2). A subset of these, massive fungating breast lesions, involve invasion of neoplastic cells through the skin, an even rarer occurrence, with no definitive epidemiological data as a reference. Furthermore, the presence of tumor penetration of the skin and chest wall presents a unique case that is rarely, if at all, reported on. Therefore, treatment for such lesions is not commonly described. The mainstay of LABC is the Madden modified radical mastectomy. Halstead's radical mastectomy, representing $0.05 \%$ of all mastectomies, is rarely performed today as it is generally reserved for lesions that invade the chest wall (3). In the absence of chest wall invasion, breast conserving therapies are greatly preferred due to favorable outcomes and fewer morbidities.

The radical mastectomy includes the removal of the entire breast tissue, both pectoralis muscles, and the entire axillary lymphatic tissue. Complications include, but are not limited to, infections, skin necrosis, paresthesias, denervation of the serratus or latissimus muscles, rib cartilage damage, pneumothoraces, and lymphedema of the arm $(3,4)$. In rare instances, local recurrence and metastasis can occur but is not robustly described in the setting of post-radical mastectomy of such invasive lesions. We describe a case of a 90-year-old female with a massive, necrotic, fungating invasive ductal carcinoma with skin and chest wall invasion that demonstrated initial response to radical mastectomy. However, her malignancy recurred in a short time frame with regional metastasis to the contralateral breast, multiple lymph nodes, and the left lower lobe of the lung. Her initial presentation, advanced age, living situation, histological tumor characteristics, and recurring metastasis present a case with no clearly established treatment. We discuss the benefit of surgical management and weigh it against other treatment options. We present the following case in accordance with the CARE reporting checklist (available at https://asj. amegroups.com/article/view/10.21037/asj-21-45/rc).

\section{Case presentation}

A 90-year-old Caucasian female presented to our surgical clinic from an oncology referral, complaining of a malodorous right breast wound. Her past medical history was negative for estrogen therapy, alcohol use, and previous radiation exposure, but positive for hypertension, congestive heart failure, coronary artery disease, and atrial fibrillation. The status of her last mammogram was unknown. The patient was oblivious to the presence of any masses or the onset of her lesion. Her ability to care for open lesion was suspect, as she admitted difficulty showering, dressing, ambulating, and finding transportation to seek medical care. Upon physical exam, a very large, hard, fixed, fungating, ulcerated, and necrotic cavitary tumor on her right breast was appreciated, with malodorous discharge originating from the necrotic center (Figure 1). This mass composed her entire right breast. Within the necrotic center, the patient had packed paper towels and bath tissues, as she was under the impression, she possessed only a leaking wound and admitted difficulty to keeping herself and clothing clean due to the copious amounts of malodorous drainage. Computed tomography scan of the chest revealed a $13 \times 9 \times 8 \mathrm{~cm}^{3}$ right breast mass demonstrating skin ulceration, central necrosis, invasion into the chest wall musculature and axillary lymphadenopathy consistent with metastasis (Figure 2). The left breast did not exhibit any suspicious masses on imaging or physical exam at the time. Medical oncology deemed the patient too frail to tolerate chemotherapy. Complicating her management were multiple social issues, poor family support, and lack of transportation. Therefore, she was not a candidate for radiation therapy, which would require strict compliance to multiple treatment sessions. The patient had already missed several appointments with our office, further indicating she did not have the means to comply with such treatment. The decision was made to bypass pre-operative core needle biopsy as it was already apparent that the mass was malignant and would not alter our management. It was decided that radical mastectomy would be the best option for the patient to alleviate her disease burden and allow her to perform her daily activities of living.

During the case, an extended elliptical incision was necessary. Oriented in a cranio-caudad direction, it 


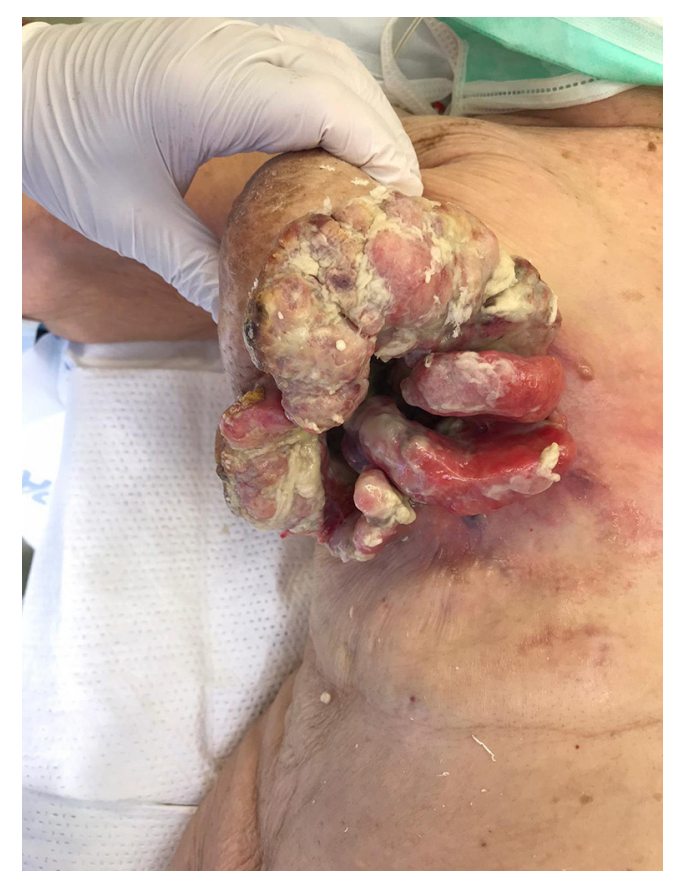

Figure 1 Pre-operative image of large, fungating, necrotic lesion of the right breast.

completely encompassed the nipple-areolar complex and tumor. To allow for closure of the flaps with as little tension as possible, an incision from the coracoid process to the umbilicus was necessary. Flaps were raised, while the skin, tumor, breast tissue, and pectoralis major and minor were removed in standard fashion, including excision of level I, II and III axillary lymph nodes. Two drains were placed. The wound was reapproximated with interrupted nylon sutures (Figure 3). Histological findings revealed grade 3 invasive ductal carcinoma (T4bN2M0) negative for estrogen receptor (ER-negative), progesterone receptor (PR-negative), and non-amplification of human epidermal growth factor receptor 2 (HER2-negative). Also included were evidence of invasion into skeletal muscle and skin. 8 out of 10 excised axillary lymph nodes were positive for metastasis.

Post-operative complications included NSTEMI, presyncope due to orthostatic hypotension, paroxysmal atrial fibrillation with rapid ventricular rate. Furthermore, mild lymphedema near the site of incision was observed. This did not require any intervention. The rest of her post-operative issues were resolved with medical management without further complications.

On postoperative day 28 , the patient was noted to have

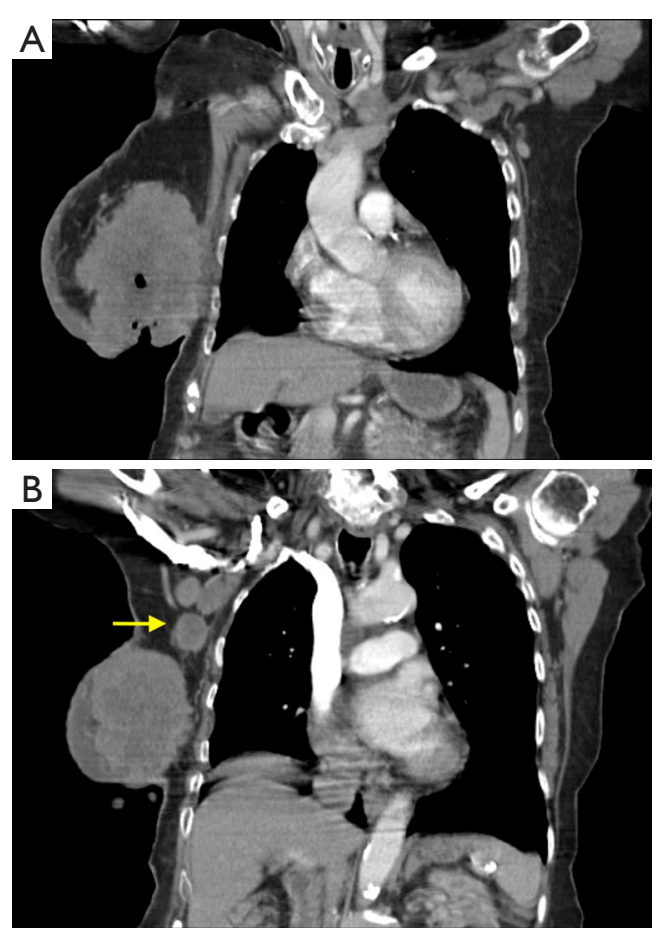

Figure 2 CT images of the right breast lesion. (A) Coronal view of right breast lesion measuring approximately $13.0 \times 9.0 \times 8.0$ $\mathrm{cm}^{3}$ evidence of skin ulceration, central necrosis, and chest wall invasion. (B) Coronal cross section exhibiting right axillary lymphadenopathy consistent with metastasis. Largest lymph node measuring approximately $2.0 \mathrm{~cm} \times 3.1 \mathrm{~cm}$ (yellow arrow).

a minimal amount of skin edge necrosis (Figure 4A). This was not completely unexpected, given that a portion of the wound closure was under some tension. This was sharply debrided and wound vacuum assisted closure (V.A.C) was placed (Figure 4B). The wound healed well without further complications.

The patient returned to clinic 4 months post-op, complaining of a lesion on the contralateral breast that had appeared precipitously. The lesion was noted in the medial aspect of the left breast, near the inframammary fold. It was noted to be superficial, tender, and raised with skin ulceration. (Figure 5). Due to her recent surgery and the concerning appearance of the lesion for a cutaneous metastasis, she was scheduled for excisional biopsy/ lumpectomy instead of performing a punch or core needle biopsy. The patient was notified that this would likely not improve survivability, but she elected for mastectomy as she desired anatomical symmetry and possible decreased risk of future cutaneous lesions. An elliptical incision was made 
surrounding the lesion to include grossly negative margins and underlying normal breast tissue. It was closed with a running vertical mattress nylon suture. Pathological analysis of the excised tissue confirmed the presence of metastasis, revealing triple negative invasive ductal carcinoma. The patient was subsequently scheduled for a simple mastectomy, as staging was considered moot at this point.

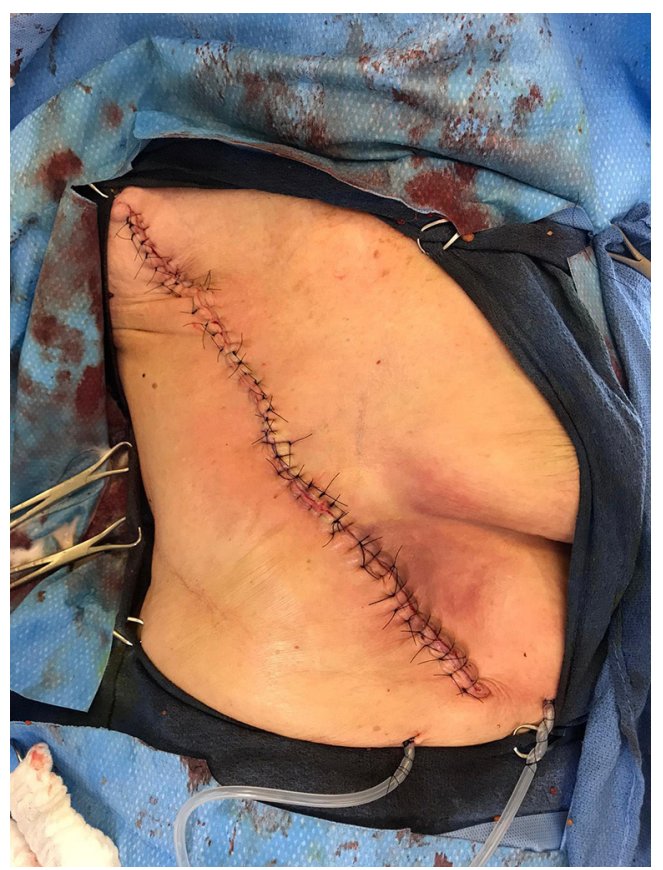

Figure 3 Approximated wound with interrupted nylon sutures after excision of breast tissue, tumor, pectoralis major and minor, and axillary lymph nodes.
However, intraoperatively the patient was noted to have multiple enlarged and hard left axillary lymph nodes. Therefore, the decision was made to convert the procedure to a modified radical mastectomy. Histological analysis revealed no additional focus of neoplastic tissue within the left breast. However, 4 of the 7 excised axillary lymph nodes were positive for metastasis.

Post operatively the patient did well and her wound healed without complication. She was discharged to home

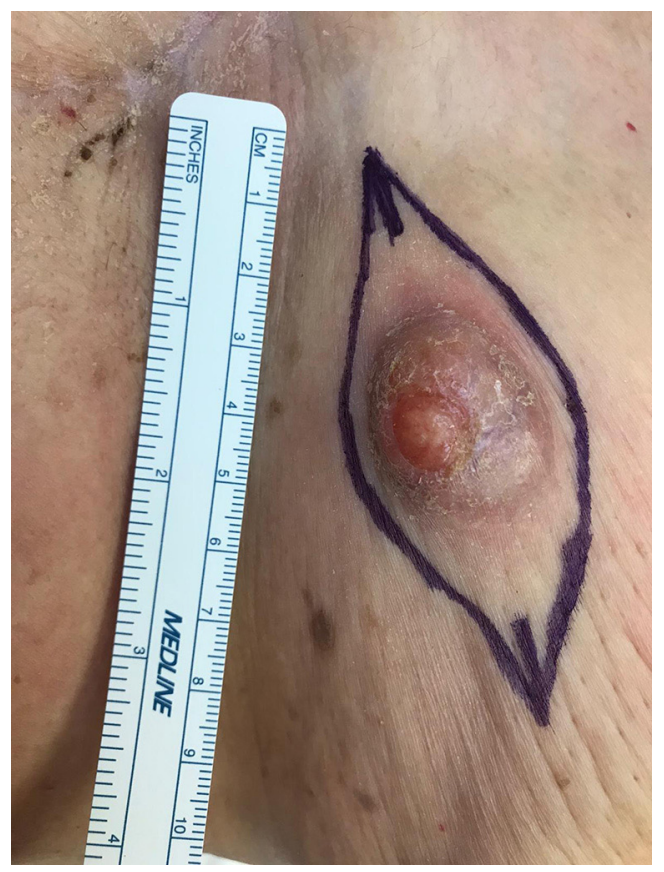

Figure 5 Superficial left breast lesion, suspicious for malignancy.
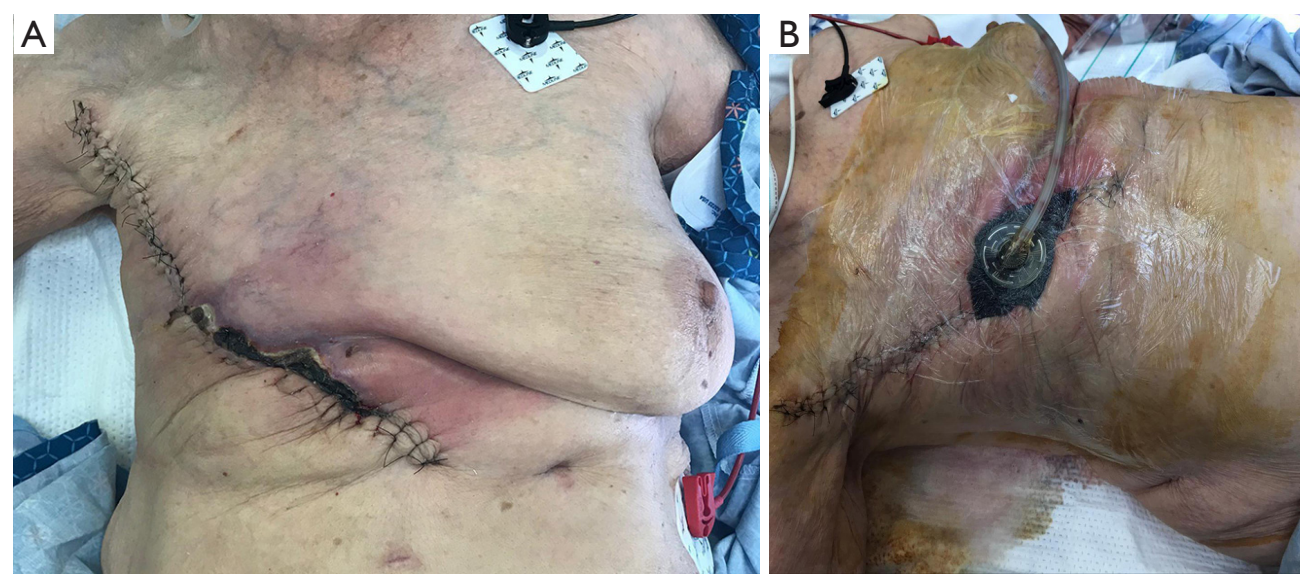

Figure 4 Postoperative complications related to closure of right radical mastectomy and its correction. (A) Skin necrosis at visible wound edges on postoperative day 28. (B) Wound vacuum assisted closure was placed. 


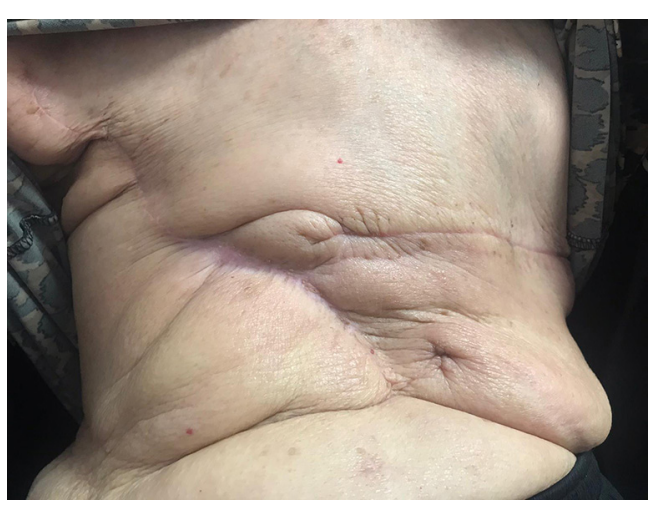

Figure 6 Post-operative image of bilateral mastectomy scars.

with home health care. After medical oncology reevaluation, it was recommended that no further treatment be given due to her advanced age, comorbidities, and triple negative status. Her post-operative scar healed without complications (Figure 6). She admitted that her ability to complete her activities of daily living were significantly improved as she did not have to carry the burden of a large, malodorous mass. However, follow-up PET-CT from skull to midthigh revealed hypermetabolic areas in the right axillary, internal thoracic, and supraclavicular nodes consistent with metastasis. A hypermetabolic nodule in the left lower lobe consistent with metastasis was also detected. The patient remains alive at the time of this writing, seven months after her initial radical mastectomy.

All procedures performed in this study were in accordance with the ethical standards of the institutional and/or national research committee(s) and with the Helsinki Declaration (as revised in 2013). Written informed consent was obtained from the patient for publication of this case report and accompanying images. A copy of the written consent is available for review by the editorial office of this journal.

\section{Discussion}

Large fungating breast lesions are rarely seen in the United States and developed countries due to advances in screening guidelines and treatment that prevent such lesions from manifesting (5). Factors hypothesized to increase the risk of such disease include low income, language barriers, psychiatric illness, decreased access to healthcare services, homelessness, and disability (6). Aside from the threats associated with malignancy, challenges in management of this patient population include chronic pain, frequent infection, malodor, social isolation, and general debilitation. Due to the limited data, there are no fully established guidelines for the management of fungating $\operatorname{LABC}(6,7)$.

Radical or modified mastectomies are commonly utilized procedures to manage fungating LABC. Complications after mastectomy are not uncommon. In this case, the patient returned with flap necrosis on post-op day 28. Flap necrosis occurs in about $8 \%$ of patients and is related to inadequate blood supply to the flap, wound excessive tension, obesity, and incision type (4). In this case, autologous flap with the latissimus dorsi would have been an acceptable closure technique, as it has proven useful for closure of salvage mastectomy with a large skin defect. However, there was no plastic surgery coverage available at this time in the small community hospital at which the patient was being cared for. Her previous inability to comply with all her appointments helped to guide our decision away from referring her to a tertiary care center for plastic surgery evaluation. Furthermore, utilizing a flap technique would have added considerable operative time, increasing her risk of morbidity. Another common complication postmastectomy includes the development of lymphedema. Axillary lymph node dissection is the most significant risk factor for the development of lymphedema, with a reported incidence of greater than 20\% (4). During the course of treatment, the patient presented with mild lymphedema after her first mastectomy that did not require any therapy.

Neoadjuvant chemotherapy can be administered for debulking. The question remains if chemotherapy would be warranted in all cases with fungating LABC. In the case with the patient presented, pathological reports confirmed the presence of triple negative breast cancer (TNBC), making hormonal targeting therapies ineffective. TNBC accounts for $10-20 \%$ of all breast carcinomas and is associated with a poor prognosis (8). Anthracycline chemotherapy with adjuvant radiation therapy is considered the first line treatment for metastatic TNBC. However, this approach demonstrates less impact with age and overall does not improve the survival benefit of patients (8). Furthermore, approximately a third of TNBC patients relapse with local recurrence or metastasis within 5 years of chemotherapy and radiation (9).

This patient presented four months after radical mastectomy with a contralateral mass that was later determined to be a metastatic lesion. While the patient did not have any serious surgical complications after her initial radical mastectomy, it did not eliminate the threat of recurrent malignancy. Likewise, the contralateral 
mastectomy did not deter the development of distant metastasis. In general, local recurrence and contralateral breast cancer rates are extremely low $(\sim 0.2-0.3 \%)(10)$. However, in this case, it was not surprising given her tumor stage and aggressive subtype. Adjuvant therapy increases survival rate by $10 \%$ in women over the age of 50 . But in older women, this number drops to approximately $3 \%$ (11). Overall, while $80 \%$ of women with metastatic breast cancer receive adjuvant chemotherapy, $40 \%$ still relapse and die (11). Therefore, there may be questionable benefits to exposing older patients to chemotherapy in cases of fungating LABC when surgery may be a suitable option. More robust studies would need to be conducted on comparing these options to substantiate this as literature comparing the outcomes of treatment of stage 4 TNBC women greater than 90 years old with multiple comorbidities is scarce. Our patient has responded well in regards to local control from radical and modified radical mastectomies. Despite the eventual development of distant metastasis, at age 90, surgical management has greatly improved her quality of life, relieving the burden of a large, malodorous, and draining tumor. Judgement of the outcome depends on the goal of management, especially in older patients, where attempts at prolonging survival may compromise their quality of life. Treatment plans that consider biopsychosocial factors to achieve a unique balance between prolonging survival, palliating symptoms, and delaying disease progression, may be more beneficial to patients given the current limitations in eradicating such disease.

\section{Acknowledgments}

Funding: None.

\section{Footnote}

Reporting Checklist: The authors have completed the CARE reporting checklist. Available at https://asj.amegroups.com/ article/view/10.21037/asj-21-45/rc

Peer Review File: Available at https://asj.amegroups.com/ article/view/10.21037/asj-21-45/prf

Conflicts of Interest: All authors have completed the ICMJE uniform disclosure form (available at https://asj.amegroups. com/article/view/10.21037/asj-21-45/coif). The authors have no conflicts of interest to declare.
Ethical Statement: The authors are accountable for all aspects of the work in ensuring that questions related to the accuracy or integrity of any part of the work are appropriately investigated and resolved. All procedures performed in this study were in accordance with the ethical standards of the institutional and/or national research committee(s) and with the Helsinki Declaration (as revised in 2013). Written informed consent was obtained from the patient for publication of this case report and accompanying images. A copy of the written consent is available for review by the editorial office of this journal.

Open Access Statement: This is an Open Access article distributed in accordance with the Creative Commons Attribution-NonCommercial-NoDerivs 4.0 International License (CC BY-NC-ND 4.0), which permits the noncommercial replication and distribution of the article with the strict proviso that no changes or edits are made and the original work is properly cited (including links to both the formal publication through the relevant DOI and the license). See: https://creativecommons.org/licenses/by-nc$\mathrm{nd} / 4.0 /$.

\section{References}

1. American Cancer Society. Breast Cancer Facts \& Figures 2019-2020. Atlanta: American Cancer Society, Inc. 2019.

2. Simos D, Clemons M, Ginsburg OM, et al. Definition and consequences of locally advanced breast cancer. Curr Opin Support Palliat Care 2014;8:33-8.

3. Jones C, Lancaster R. Evolution of Operative Technique for Mastectomy. Surg Clin North Am 2018;98:835-44.

4. Goethals A, Rose J. Mastectomy. In: StatPearls [Internet]. Treasure Island (FL): StatPearls Publishing, 2021.

5. Bichoo RA, Yadav SK, Mishra A, et al. Fungating Breast Cancer: Experience in Low and Middle Income Country. Indian J Surg Oncol 2020;11:281-6.

6. Sood A, Daniali LN, Rezzadeh KS, et al. Management and Reconstruction in the Breast Cancer Patient With a Fungating T4b Tumor. Eplasty 2015;15:e39.

7. El Saghir NS, Eniu A, Carlson RW, et al. Locally advanced breast cancer: treatment guideline implementation with particular attention to low- and middle-income countries. Cancer 2008;113:2315-24.

8. Al-Mahmood S, Sapiezynski J, Garbuzenko OB, et al. Metastatic and triple-negative breast cancer: challenges and treatment options. Drug Deliv Transl Res 2018;8:1483-507. 
9. Wright HJ, Hou J, Xu B, et al. CDCP1 drives triplenegative breast cancer metastasis through reduction of lipid-droplet abundance and stimulation of fatty acid oxidation. Proc Natl Acad Sci U S A 2017;114:E6556-65.

10. Euhus DM. Choosing Bilateral Mastectomy. Breast J

doi: 10.21037/asj-21-45

Cite this article as: $\mathrm{Ng} \mathrm{C}$, Jamieson NC, Spinale RC. Radical mastectomy of massive stage IV triple negative breast cancer to improve hygiene and comfort in a 90-year-old woman: a case report. AME Surg J 2022;2:19.
2016;22:605-6.

11. Weigelt B, Peterse JL, van 't Veer LJ. Breast cancer metastasis: markers and models. Nat Rev Cancer 2005;5:591-602. 